\title{
$\mathrm{PH} 103_{\text {actualidad }}$
}

\section{Las XX jornadas de la AIP reflexionan sobre el turismo oscuro y los espacios de memoria traumática}

El sector turístico es una actividad que se renueva constantemente. La oferta se diversifica al mismo tiempo que las motivaciones de la demanda. El subsector del turismo cultural es uno de los que mejor acredita esto. La cultura y el patrimonio son fuente ilimitada de recursos. Aquellos que están en auge en la actualidad son los que están relacionados con hechos o experiencias traumáticas de una sociedad o de la propia humanidad. En esta contribución se presentan las XX Jornadas para la Interpretación del Patrimonio, celebradas en el mes de marzo y donde se debatieron las tipologías de este turismo "oscuro", sus públicos, motivaciones, metodologías para la puesta en valor e interpretación y sus dilemas ético-morales.

Óscar Navajas Corral | Dpto. Historia y Filosofía, Universidad de Alcalá

Patricia Muñoz Raña | Asociación para la Interpretación del Patrimonio (AIP)

URL de la contribución <http://www.iaph.es/revistaph/index.php/revistaph/article/view/4889>

Tradicionalmente, el turismo ha estado relacionado con el entretenimiento y con motivaciones positivas (Beech 2009). No obstante, desde finales de los años 90 se han ido consolidando tipologías relacionadas con la visita a espacios donde la motivación es el sufrimiento, el miedo, la tristeza, el dolor e incluso la muerte. Esta actividad ha ido evolucionando desde un discurso fundamentado en descripciones históricas e impactos negativos hasta conseguir generar activaciones patrimoniales con una visión transversal del pasado e interconectada con el presente y con el futuro. Es decir, ya no son únicamente testimonios de un pasado oscuro (traumático), sino también generadores de emociones, reflexiones y comportamientos positivos y, por supuesto, de "peregrinación" turística.

El desarrollo de este subsector de la industria turística ha tenido, hasta la fecha, una aparente repercusión limitada en nuestras fronteras. Sin embargo, la realidad es diferente. Las XX Jornadas para la Interpretación del Patrimonio, organizadas el pasado mes de marzo por la Asociación para la Interpretación del Patrimonio (AIP), con la colaboración de la Universidad de Alcalá, constataron esto.

El punto de partida fue la paradoja existente entre la relación del turismo como una actividad asociada al ocio y a lo positivo y unos patrimonios y espacios de donde

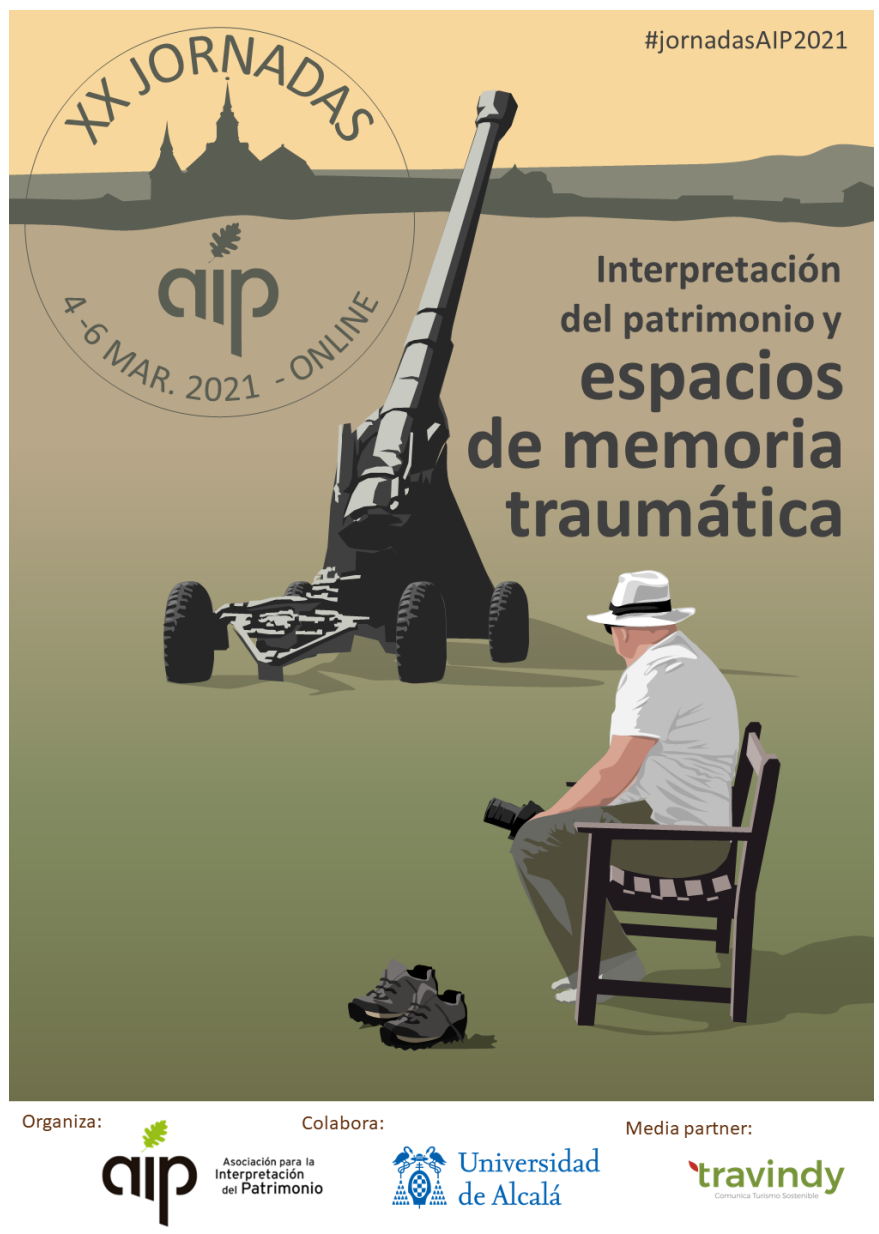


emanan intangibles como dolor, miedo, trauma, ira, venganza, injusticia, muerte, etc. En este contexto, surgían preguntas como: ¿podemos entender que la visita a lugares donde han ocurrido hechos traumáticos esté asociada a una actividad basada en el ocio?, ¿qué motivaciones conducen a la ciudadanía a visitar estos espacios?, ¿qué metodologías resultan más efectivas para poner en valor este acervo patrimonial? o ¿dónde están los límites, la ética y la moral en los procesos de patrimonialización del sufrimiento humano?

El primer paso para contestar a estas cuestiones fue analizar el propio concepto de turismo oscuro (Dark Tourism). El turismo oscuro es un denominador común para un cajón de sastre donde se pueden incluir desde itinerarios espectrales, visitas a museos de la inquisición, campos de concentración, campos de batalla, museos militares, catástrofes naturales, etc., hasta visitas a países con actuales conflictos armados ${ }^{1}$, escenarios de películas y series ${ }^{2}$ o a barrios con necesidades y problemáticas sociales ${ }^{3}$.

Cada uno de estos patrimonios y espacios con un contenido relacionado con el terror, el sufrimiento o la tragedia se pueden jerarquizar en función de la intensidad de su "oscuridad" (Seaton 1996; Stone 2006); es decir, de la cercanía del hecho a cada visitante o sociedad. Las inter-

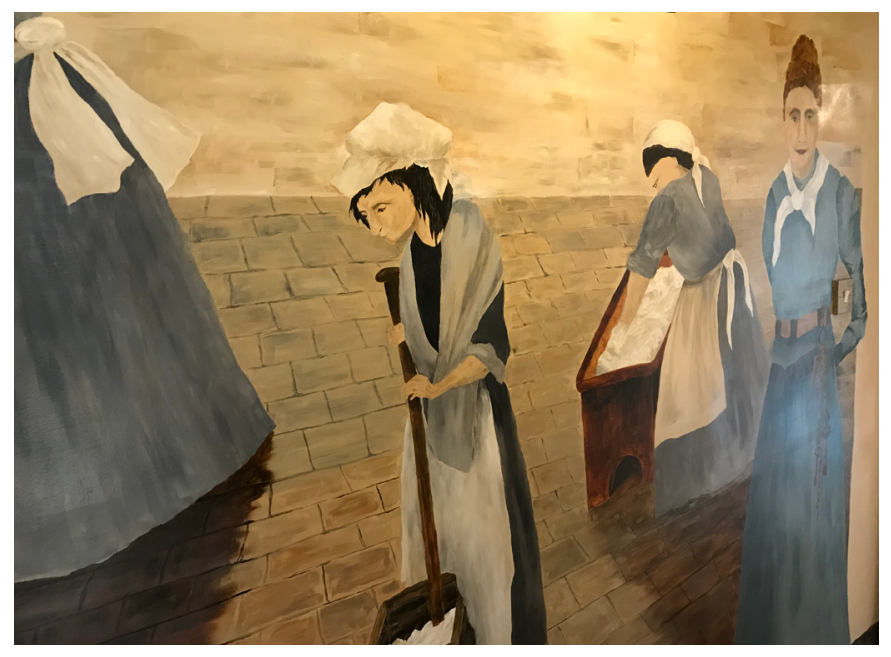

Correccional de mujeres, uno de los anteriores usos del Wymondham Heritage Museum (Reino Unido) | foto Óscar Navajas Corral venciones en esta sesión de las jornadas constataron cómo la visita a este tipo de espacios está modificando nuestra tradicional vinculación con los hechos traumáticos e incluso con la muerte, en una nueva relación que se ha denominado como "tanacapitalismo" (Korstanje 2017).

La segunda sesión tenía como objetivo conocer qué es lo que mueve a las personas a dedicar su tiempo de ocio a visitar una memoria traumática. En las diferentes presentaciones las motivaciones más recurrentes estaban relacionadas con el conocimiento, la educación, la identidad, la rememoración o la simple curiosidad. Junto a ellas, también se planteó la existencia de alicientes directamente conectados con elementos de conflicto, violencia, sadismo o la propia vinculación con la muerte.

La forma de afrontar estas motivaciones -y los públicos- a la hora de poner en valor estos patrimonios y sus espacios se trató en la tercera sesión. Se abordaron metodologías como la Hot Interpretation (Uzzell 1989), la técnica de muchas voces (Ryland 2017), la Historia Pública (Cauvin 2020) o la Curva Inzovu (Pratley 2016). Estas y otras metodologías ponen el acento en que las activaciones patrimoniales deben estar dirigidas a la parte emocional más que a la cognitiva, fomentando las competencias sensitivas y emocionales. También se reseñó la importancia de incorporar la inclusión y la participación del público y de las comunidades en la construcción de las narraciones.

Si por algo se caracterizan las jornadas anuales de la AIP es por ser espacios de participación y debate. Con una temática como la que se abordaba en esta edición era de esperar que surgieran numerosas cuestiones, reflexiones y lides. Algunas de las más relevantes estuvieron enfocadas en las posibilidades para el futuro de las sociedades que posee la puesta en valor de estos patrimonios, el impacto que este tipo de turismo tiene, o puede tener, en los núcleos rurales; las acciones participativas como metodología para su activación, el diálogo y el (re)planteamiento sobre cuestiones éticas; el concepto de "objetividad", la instrumentalización de estos bienes y sus memorias o los procesos de socialización y participación con patrimonios y memorias tan vivas aún en la sociedad. 


\section{Mural de cultura crítica.}

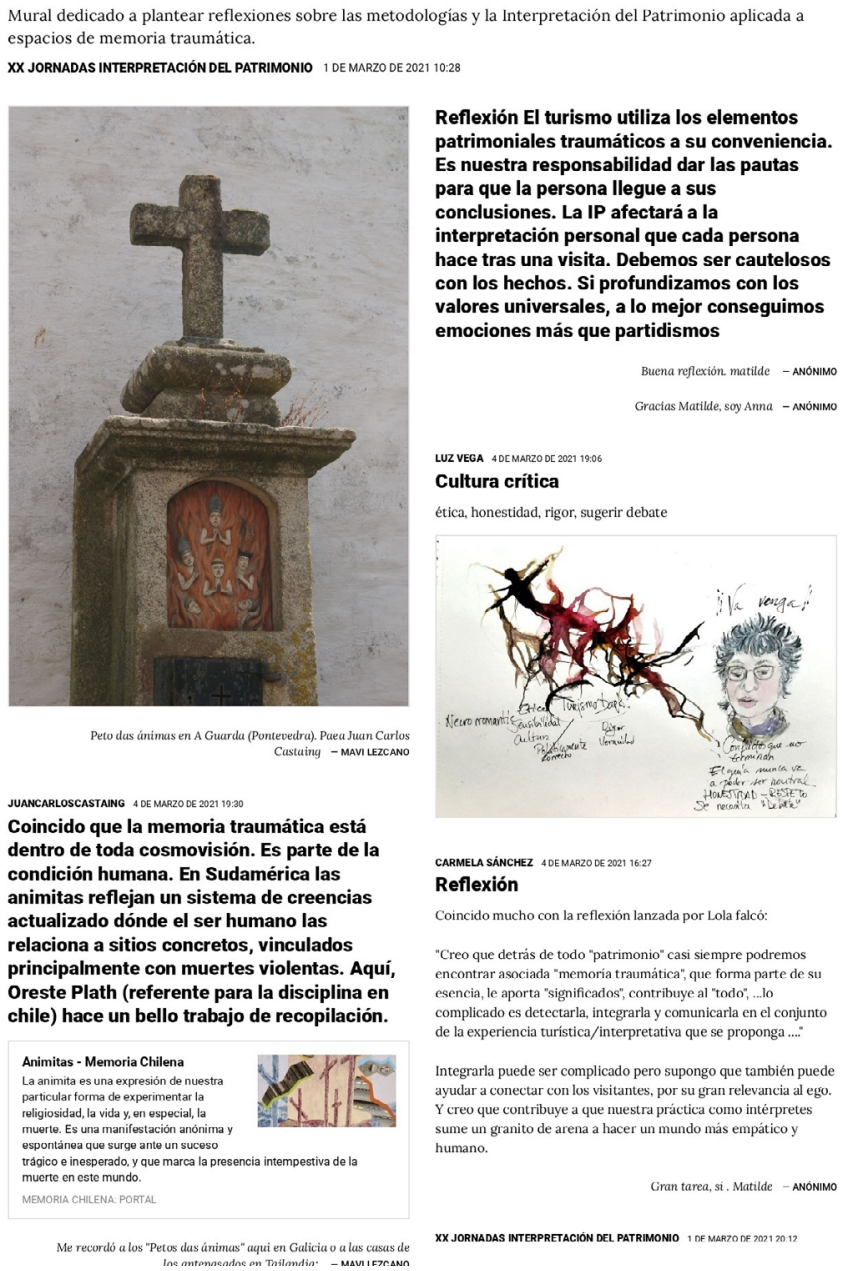

Mural Cultura Crítica | fuente Asociación para la Interpretación del Patrimonio

\section{NOTAS}

1. Empresas como WarZone Tours realizan viajes a Sudán y Gaza; Untamed Borders, a Afganistán, Corea o Etiopía; o Political Tours realizaba a Kósovo e Irán.

\section{Series como Narcos se han convertido en un fetiche} para los turistas. Se ha llegado a denominar como "narcoturismo".

3. Se está desarrollando una industria turística en torno a barrios empobrecidos como las favelas o distritos de la India (Martínez 2019).

\section{BIBLIOGRAFÍA}

- Beech, J. (2009) Genocide tourism. En: Sharpley, R. y Stone, P. (ed.) The darker side of travel: The theory and practice of dark tourism. Bristol: Channel View Publications, pp. 207-223

- Cauvin, T. (2020) Campo nuevo, prácticas viejas: promesas y desafíos de la historia pública. Hispania Nova, n. ${ }^{0} 1$, Extraordinario, pp. 7-51. Disponible en: https://e-revistas.uc3m. es/index.php/HISPNOV/article/view/5365 [Consulta: 10/01/20 21]

- Korstanje, M. (2017) Towards New Horizons in Dark Tourism Studies. En: Korstanje, M. y Handayani, B. (ed.) Gazing at Death. Dark Tourism as an Emergent Horizon of Research. New York: Nova Science Publishers, pp. 1-14

- Martínez, A. (2019) El gueto que noqueó al Taj Mahal. El País, 21 de agosto de 2019. Disponible en: https://elpais. com/cultura/2019/08/20/actualidad/1566308413_748005.html [Consulta: 12/04/2021]

- Pratley, C. (2016) The Inzovu Curve. Interpretation Journal, Association for Heritage Interpretation, vol. 21, n. $^{0}$ 2, pp. 28-29. Disponible en: https://www.jiscmail.ac.uk [Consulta: 20/02/2021]

- Ryland, P. (2017) Reporting Research 3-Hot interpretation. Interpretation Journal, Association for Heritage Interpretation, vol. 22, n. ${ }^{\circ} 1$, pp. 5-6. Disponible en: http://eprints. bournemouth. ac.uk/29154/3/AHI.2017. Reporting\%20research3. Hotinter pretation.v2.pdf [Consulta: 08/09/2020]

- Seaton, A. (1996) Guided by the dark: From thanatopsis to thanatourism. International Journal of Heritage Studies, vol. 2, n. ${ }^{\circ} 4$, pp. $234-244$

- Stone, P. (2006) A dark tourism spectrum: Towards a typology of death and macabre related tourist sites, attractions and exhibitions. Tourism, vol. 54, n. ${ }^{\circ}$ 2, pp. 145-160. Disponible en: http://clok.uclan.ac.uk/27720/1/27720\%20fulltext_stamped.pdf [Consulta: 15/06/2020]

- Uzzell, D. (1989) The Hot Interpretation of War and Conflict. En: Uzzell, D. L. (ed.) Heritage Interpretation: The Natural and Built Environment, vol. 1. London and New York: Belhaven Press, pp. 33-47 